\title{
Almacenamiento de carbono en el suelo del Bosque Natural Cayo Quemado. Livingston, Izabal, Guatemala
}

\author{
Jeff Hendrik Taque Aroche \\ Epesista de la carrera de Gestión Ambiental del Centro Universitario \\ de Izabal de la Universidad de San Carlos de Guatemala \\ jehendrik@gmail.com \\ Eddi Alejandro Vanegas Chacón \\ Doctor Profesor de la Facultad de Agronomía de la Universidad \\ de San Carlos de Guatemala vanegaseddi@gmail.com
}

Fecha de recepción: 17/07/2017 Fecha de aceptación: 15/08/2017

\begin{abstract}
Resumen
Los ecosistemas marino costeros prestan servicios ambientales de invaluable importancia, por lo que a nivel internacional de forma incipiente se están elaborando políticas para su conservación a través de incentivos económicos que beneficien a los lugareños que interactúan con estos ecosistemas. La aldea Cayo Quemado, ubicada en Livingston, Izabal, cuenta con un bosque natural con predominancia de Mangle rojo (Rizophora mangle) 38.14 \%; Zapotón (Pachira acuatica) 19.07 \%; Cahué (Pterocarpus officinalis) $18.56 \%$ y Anonillo (Rollinia pittieri), $7.22 \%$; del cual se carece de información para realizar un inventario de captura de carbono tanto del epigeo como del suelo y raíces. Mediante la colecta de muestras de suelos (sin raíces) en el estrato 0-30 cm; se determinó en laboratorio el contenido de materia orgánica, con este estudio se muestra que existe efecto de especie vegetal y posición sobre la captura de carbono en el suelo, donde el sistema manglar almacena menor cantidad en relación con las otras tres especies (no habiendo diferencias estadísticas entre ellas). No obstante, a nivel de la extensión del Cayo Quemado, el Mangle capta 0.6576, el Zapotón 0.5446, el Cahué 0.5009 y el Anonillo 0.1912 para un total de 1.89 Mg $\mathrm{CO}_{2}$ en 10.56 ha de bosque natural.
\end{abstract}

\section{Palabras clave}

Gases efecto invernadero, captura de carbono, materia orgánica, mangle, ecosistemas marino costeros.

\begin{abstract}
Coastal marine ecosystems provide invaluable environmental services, thereford at international level, policies for their conservation are being developed in an incipient way through economic incentives that benefit the villagers who interact with these ecosystems. Cayo Quemado, in Livingston, Izabal has a natural forest with predominance of Red Mangrove (Rizophora mangle) 38.14 \%; Zapotón (Pachira acuatica) 19.07 \%; Cahue (Pterocarpus officinalis) $18.56 \%$ and Anonillo (Rollini apittieri), $7.22 \%$. About it, there is a lack of information to perform an inventory of carbon capture of both the epigeal and the soil and roots. By collecting soil samples (without roots) in the level 0-30 cm; at laboratory the organic matter content was calculated, this researchdetermined that there is an effect of plant species and position on the carbon capture in the soil, where the mangrove system stored less quantity in relation to the other three species (there being no statistical differences between them). However, at the level of the Cayo Quemado territorial extension, the Mangrove captured 0.6576, Zapotón 0.5446; Cahué 0.5009 and Anonillo 0.1912 to add a total of $1.89 \mathrm{Mg} \mathrm{CO}_{2}$ in 10.56 ha of natural forest.
\end{abstract}

\section{Key words}

Greenhouse gases, carbon capture, organic matter, mangrove, coastal marine ecosystems. 


\section{Introducción}

Los ecosistemas de mangle se localizan en estuarios tropicales y subtropicales, los cuales constituyen la interfase entre comunidades terrestres y marinas, que interactúan a diario con las dinámicas oceánicas (FAO, 2007). Los ecosistemas asociados a los manglares generan una serie de productos y servicios a los pobladores lugareños, como a nivel de biota asociada al ecosistema y como almacenador de dióxido de carbono (Lozano, 2007). Los manglares revisten de importancia por sus funciones ecológicas como filtros naturales, refugio y hábitat para una diversidad de organismos acuáticos y terrestres que dependen de lo que el manglar produce, dinámica del ciclo del carbono, cuya productividad está entre las mayores de los ecosistemas costeros (Lacerda et al. 2001). Sin embargo, las actividades humanas y algunos factores ambientales han provocado un estado crítico en estos, alrededor del mundo (Castellanos \& Menjívar, 2011). Se estima que en los últimos veinte años del siglo XX se perdió $25 \%$ de la superficie de manglares en el mundo (Wilkie y Fortuna, 2003). El ecosistema de mangle lo constituyen los árboles de mangle, especies arbustivas asociadas, otras plantas, hongos, bacterias, microbios, animales y distintos factores abióticos (Blanco, Ortiz, \& Urrego, 2015). En 1971, se estableció el tratado intergubernamental RAMSAR de los humedales, que enfatiza la conservación y uso racional de los recursos naturales. Guatemala se adhirió a este tratado el 26 de enero de 1988, a través del decreto legislativo 4-88 del Congreso de la República (CONAP\& FONACON, 2015). Los bosques de manglar son los principales reservorios de biomasa aérea y carbono aéreo en las zonas marino-costeras tropicales y subtropicales (Adame et al. 2013), no obstante, el suelo puede actuar como fuente o reservorio de C dependiendo de su uso y manejo (Lal, 1997; Lozano, 2007). En manglares existe evidencia del rol de la captura de carbono en el suelo (parte mineral + raíces); siendo inclusive en la exuberancia del sistema mayor a aquel almacenado en el epigeo (Murdiyaso et al. 2009; Donato et al. 2011; BIOMARCC-SINAC-GIZ, 2012). El bosque natural de la aldea Cayo Quemado, Livingston, Izabal, cuenta con una extensión de 12.69 ha. En este bosque predomina el mangle rojo (Rizophora mangle) 38.14 \%; el Zapotón
(Pchira acuatica) $19.07 \%$; el Cahue (Pterocarpus officinalis) $18.56 \%$, el Anonillo (Rollini apittieri) $7.22 \%$ y otras especies $17.01 \%$; con múltiples fines ecológicos (CONAP-INAB, 2014). La estabilidad ecológica de este Cayo depende de la conservación tanto del mangle como de las especies vegetales asociadas en forma natural. Con objeto de establecer la importancia de las interacciones del bosque natural Cayo Quemado, esta investigación evaluó el efecto de las especies vegetales predominantes sobre la captura de carbono en el suelo; como indicador biológico del sistema Manglar.

\section{Referente teórico}

El aumento de dióxido de carbono $\left(\mathrm{CO}_{2}\right)$ en la tierra es una preocupación a nivel mundial, ya que es considerado como uno de los gases que intervienen en el calentamiento global. Las manifestaciones del calentamiento global se reflejan en el incremento de la temperatura y cambios en el recurso hídrico, lo que reduce la estabilidad y reserva del carbono orgánico en los suelos, alterando los ciclos geobioquímicos, tal el ciclo del carbono, y esto a su vez la biomasa, diversidad biológica y medio ambiente (Martel y Cairampoma, 2012). En Guatemala, lo anterior, es la razón de ser del decreto legislativo 7-2013, objetivo de la ley marco para regular la reducción de la vulnerabilidad y la adaptación obligatoria, ante los efectos del cambio climático y la mitigación de gases efecto invernadero (Guatemala, 2013). En ese sentido, la conservación de la cobertura vegetal es de suma importancia debido a que las plantas respiran $\mathrm{CO}_{2}$ y liberan oxígeno $\left(\mathrm{O}_{2}\right)$ durante su proceso fotosintético, poseen la característica de almacenar carbono por largos periodos, por lo que se les considera reservas naturales de carbono (Schlesinger y Andrews, 2000). Según la FAO (2007) el carbono en el suelo (COS), es un elemento esencial en el ciclo del carbono, ocupando 69.8 \% del carbono de la biosfera. La forma de localizar el carbono orgánico en el suelo, es a través de residuos orgánicos poco alterados de vegetales, animales, microorganismos y humus. El carbono orgánico es el resultado de un balance entre la incorporación de materia orgánica fresca al suelo y la salida del carbono a la atmósfera en 
forma de dióxido de carbono (Martínez, Fuentes y Acevedo 2008). La materia orgánica del suelo (MOS), se puede diferenciar porque una parte es lábil, y el resto mantiene sus características químicas en relación con el material de origen: hidratos de carbono, ligninas, proteínas, tanino, ácidos grasos, ácidos húmicos y materia orgánica, que en su conjunto constituyen la calidad físico químicas del humus (Galantini, 2002; Aguilera, 2000).

\subsection{Ecosistema Manglar en Guatemala}

Los manglares son comunidades costeras dominadas por especies de árboles o arbustos que tienen la peculiaridad de crecer en aguas saladas o salobres. Son sistemas únicos de generación y almacenamiento de biomasa vegetal y refugio para especies terrestres y acuáticas. Las áreas remanentes están fuertemente amenazadas por distintas actividades humanas, tales como utilización del mangle como leña y material de construcción; cambio de uso del suelo por actividades industriales como construcción de salineras, camaroneras, infraestructura turística y finalmente crecimiento urbano desordenado (CONAP-INAB, 2014). Para el 2013, el informe técnico del estudio de la cobertura de mangle en la República de Guatemala, indicó como resultado de la investigación, revisión y análisis de imágenes satelitales, que el área total de mangle era de 18,867.08 ha desglosadas de la siguiente manera: Costa del Pacífico 17,670.56 ha y Costa del Caribe 1,196.52 ha. Siendo significativamente mayor el área de mangle en la Costa Pacífica. Esto posiblemente se debe a que la salinidad de la primera es más alta. Otro dato importante es la cantidad de hectáreas por tipo de mangle, siendo el Mangle Rojo (Rhizophora mangle) la mayor con 11,988.41 ha, seguido por el Mangle Blanco (Laguncularia racemosa) con 6,206.07 ha. En la Costa del Caribe se pudo observar la presencia de Mangle Blanco, Negro y Rojo y una especie denominada por los lugareños como Mangle Rojo Enano. Desglosándose de la siguiente manera: Mangle Rojo 1,131.06 ha, Mangle Blanco 37.20 ha y Mangle Negro 1.26 ha; enfatizando que en el área denominada Golfete, solo existen 306.64 ha de Mangle Rojo (PNUMA, 2013).

\subsection{Importancia del ecosistema manglar}

Estos ecosistemas tienen flora y fauna compleja asociada con los manglares creando ambientes altamente diversos. Los manglares juegan un rol importante en la ecología de las costas tropicales y proporcionan muchos bienes y servicios para las poblaciones humanas. Estos incluyen: protección y estabilización de la línea de costa, criaderos para numerosos recursos pesqueros económicamente importantes, y una variada fuente de productos a las poblaciones humanas costeras en forma de madera, leña y carbón. Al mismo tiempo, los manglares exportan materia orgánica que es el alimento directo de diversos recursos pesqueros o estimulante de la producción primaria en el ecosistema acuático adyacente. Sustentan importantes pesquerías tropicales porque ofrecen refugio y alimento en las etapas críticas de los ciclos de vida de muchos peces, crustáceos y moluscos, que utilizan los manglares como áreas de reproducción y crianza. En la zona costera, los manglares reducen la erosión atenuando los efectos de olas y corrientes, ofrecen protección a los cambios climático-meteorológicos e hidrodinámicos, y son refugio de los depredadores a la variada flora y fauna que coexiste en el ecosistema (Jiménez,1999).

\subsection{Deterioro e impacto ambiental de los ecosistemas manglares}

Los sistemas manglares son afectados a diario por la denominada época antropogénica (Lugo, Mediana y McGinley, 2014) definida como la época de la dominación humana sobre el mundo. Se hace necesario tener una visión clara sobre las leyes, políticas, acuerdos internacionales y las acciones locales que se ocupan de la conservación de los bosques de mangle en el trópico y subtrópico, donde, la colaboración entre gobiernos, organizaciones no gubernamentales y las comunidades que dependen de los manglares para su sustento, es de suma importancia. La solución a la persistencia de manglares en el Antropoceno no es aislar a los manglares de la gente, sino regular las interacciones entre los manglares y los seres humanos, a través de una gestión eficaz, a través de planes de manejo sostenible implemen- 
tados por los propios pobladores locales. También es necesario ampliar el alcance del análisis ecológico de los ecosistemas de manglar para incluir las fuerzas sociales convergentes en los manglares a través de un enfoque, en la llamada Ecología Social (Saenger, 2002; Saint-Paul, 2006)

\subsection{Funciones y beneficios del manglar}

En Centroamérica y el Caribe, los humedales proveen gran cantidad de beneficios y funciones tanto al mundo natural como a los lugareños. Esto se debe a las diferentes interacciones que se desarrollan dentro del ecosistema que por años ha proporcionado servicios ambientales y fortalecido las economías de los pobladores a nivel local. Entre otros, se puede mencionar el uso de los sustratos para fines de fertilización de cultivos anuales y hortalizas, las zonas para crianzas de peces y cangrejos, la elaboración de cabos en la manufactura de escobas; ya en los aspectos ambientales, los filtros de flujo de corrientes, la protección contra tempestades, y el almacenamiento de carbono en material vegetal y suelo (URL-IARNA, 2012).

\subsection{El Cayo Quemado, Livingston, Izabal}

La aldea Cayo Quemado pertenece al municipio de Livingston, departamento de Izabal, se encuen- tra ubicado al norte, a 40 minutos de la cabecera municipal y su forma de acceso es por vía acuática, cuenta con un bosque natural de 12.69 ha, está legalmente reconocida por la municipalidad de Livingston y el Consejo Comunitario de Desarrollo (CONAP-INAB, 2014).

\subsection{Características climáticas de Cayo Quemado}

Según la clasificación de Thornthwaite de 1948, se encuentra en la zona climática de la Franja Transversal del Norte y Costa Caribe, con lluvia promedio anual de 1,800 $\mathrm{mm}$ con mayor frecuencia en junio a octubre, temperatura media anual $27{ }^{\circ} \mathrm{C}$. Para esta zona, la estación seca no es bien definida, una de sus características es que varía de muy húmedo a húmedo (CONAP, 2015).

\subsection{Especies vegetales predominantes en Cayo Quemado}

Según CONAP-INAB (2014) una de las principales características del Bosque natural es que cuenta con diferentes especies asociadas, donde las especies predominantes y que en conjunto tienen el $82.99 \%$ del área total, son Mangle rojo, (Rizophora mangle) Zapotón (Pachira acuatica), Cahue (Pterocarpus officinalis) y Anonillo (Rollinia pittieri), Tabla 1.

Tabla 1

Especies predominantes en Cayo Quemado

\begin{tabular}{|c|c|c|c|}
\hline No. & Especie & Nombre Científico & $\%$ de abundancia \\
\hline 1 & Mangle Rojo & Rizophora mangle & 38.14 \\
\hline 2 & Zapotón & Pachira acuatica & 19.07 \\
\hline 3 & Cahué & Pterocarpus officinalis & 18.56 \\
\hline \multirow[t]{2}{*}{4} & Anonillo & Rollini apittieri & 7.22 \\
\hline & Total & & 82.99 \\
\hline
\end{tabular}

Fuente: adaptado de CONAP-INAB (2014). 


\section{- $\quad$ Mangle Rojo (Rizophora mangle)}

Árbol perennifolio, se desarrolla en hábitat halófitos, salobregos y dulces, alcanza una altura hasta de $30 \mathrm{~m}$ y diámetro a la altura del pecho de hasta $50 \mathrm{~cm}$, presenta una copa redondeada, hojas simples, elípticas u oblongas, concentradas en las puntas de las ramas y alcanzan largo de $13 \mathrm{~cm}$ con un ancho hasta $5.5 \mathrm{~cm}$ presentando aspectos coriáceas, lisas, gruesas de color verde oscuro en el haz y amarillentas con puntos negros en el envés. Su flor es actinomorfa con color blanco amarillento. Su fruto es rojizo de 2 a $3 \mathrm{~cm}$ de largo. A su semilla se le denomina pro pábulos, tiene la característica que germina dentro del fruto (viviparidad), generalmente son curvos y de color verde, alcanzan un largo de 22 a $40 \mathrm{~cm}$, y pesan aproximadamente $50 \mathrm{~g}$. Su raíz se denomina fulcrea porque surgen del tallo por encima del suelo conocidas como neumatóforos (raíces aéreas que están presentes en plantas que viven en el agua, estas favorecen a la oxigenación de las partes de la planta), son ramificadas, curvas o arqueadas. Sus usos, artesanal, para elaboración de carbón, y en algunos casos de uso maderable (INAB, 1998).

\section{- Zapotón (Pachira acuatica):}

Esta especie es común en los trópicos de América, es un árbol que alcanza una altura hasta de $90 \mathrm{~m}$, con tronco grueso y ramificación monopodial verticilada, con grupos de hojas que crecen en conjunto y todas a un mismo nivel, que al estar adultos se convierten en simpodial. Tiene hojas digitadas óseas que son hojas compuestas que nacen de un peciolo común, separadas y toman forma de una mano, de 4 a 7 foliolos, de forma elíptica teniendo hasta $15 \mathrm{~cm}$ de largo (Ospina, 2010).

\section{- Cahué (Pterocarpus officinalis)}

Es un árbol que alcanza una altura promedio de $40 \mathrm{~m}$, de 60 a $90 \mathrm{~cm}$ de diámetro a altura de pecho, madera muy liviana, látex color rojizo, hojas grandes alternas pinadas impares y una vaina plana larga, redonda y alada. Es usada para elaborar carbón, la especie predomina en comunidades pantanosas incluyendo lugares con agua dulce, salobrega y salinas, se caracteriza por su rebrote vigoroso, es de suma importancia para el funcionamiento de los ecosistemas costero marinos (CONAP, 2011).

\section{- Anonillo (Rollinia pittieri)}

Este árbol presenta una altura de 15 a $30 \mathrm{~m}$, hojas simples, dísticas, es decir crecen a ambos lados del tallo formando dos filas en lados opuestos, de 10 a $18 \mathrm{~cm}$ de largo, con un ancho de 3.5 a $5 \mathrm{~cm}$, la morfología de sus hojas es oblongas de color blaquecina o grisácea en el envés, y frutos agregados sincárpicos (Zamora, 2011).

\subsection{Serie de suelos}

Esta área se desarrolló sobre rocas calcáreas, se puede encontrar depósitos marinos con relieves casi planos que corresponden a la serie Chocón, y suelos calizos con formaciones Kársticas correspondientes a la serie Chacalté, según el sistema taxonómico de Simmons, Tarano y Pinto (1959); que corresponden a suelos Inceptisoles (udepts) y Alfisoles (Udalfs) respectivamente según el USDA (2014).

\subsection{Características fisiográficas y geológicas}

Son humedales planos de la región de la depresión de Izabal, del periodo Terciario Superior Oligoceno-Mioceno; presenta rocas sedimentarias clásticas, lutitas, areniscas y conglomerados de las Formaciones Subinal conocidas como capas rojas, meandros y lagunas abandonadas. Comprende la Tinta, Panzós y Sepur, a lo largo del Polochic, Alta Verapaz; entorno norte del Lago de Izabal, El Estor, Castillo de San Felipe y Mariscos, Izabal. Los tipos de roca son relleno carbonatado, fracciones de arena, limo y arcilla de rocas metamórficas y sedimentarias, sobre sedimentos clásticos (CONAP, 2015). 


\section{Metodología}

\subsection{Delimitación del área de estudio}

Se delimitó el área de estudio mediante el uso de fotografías aéreas, con objeto de establecer el área del bosque natural a escala de semidetalle. Se delimitaron áreas de por lo menos las cuatro especies predominantes de dicho bosque: sistema de mangle; áreas de Zapotón; Cahué y Anonillo, se verificó el porcentaje de cobertura por unidad de área, Fi-

Figura 1

\section{Puntos de muestreo por bloque}

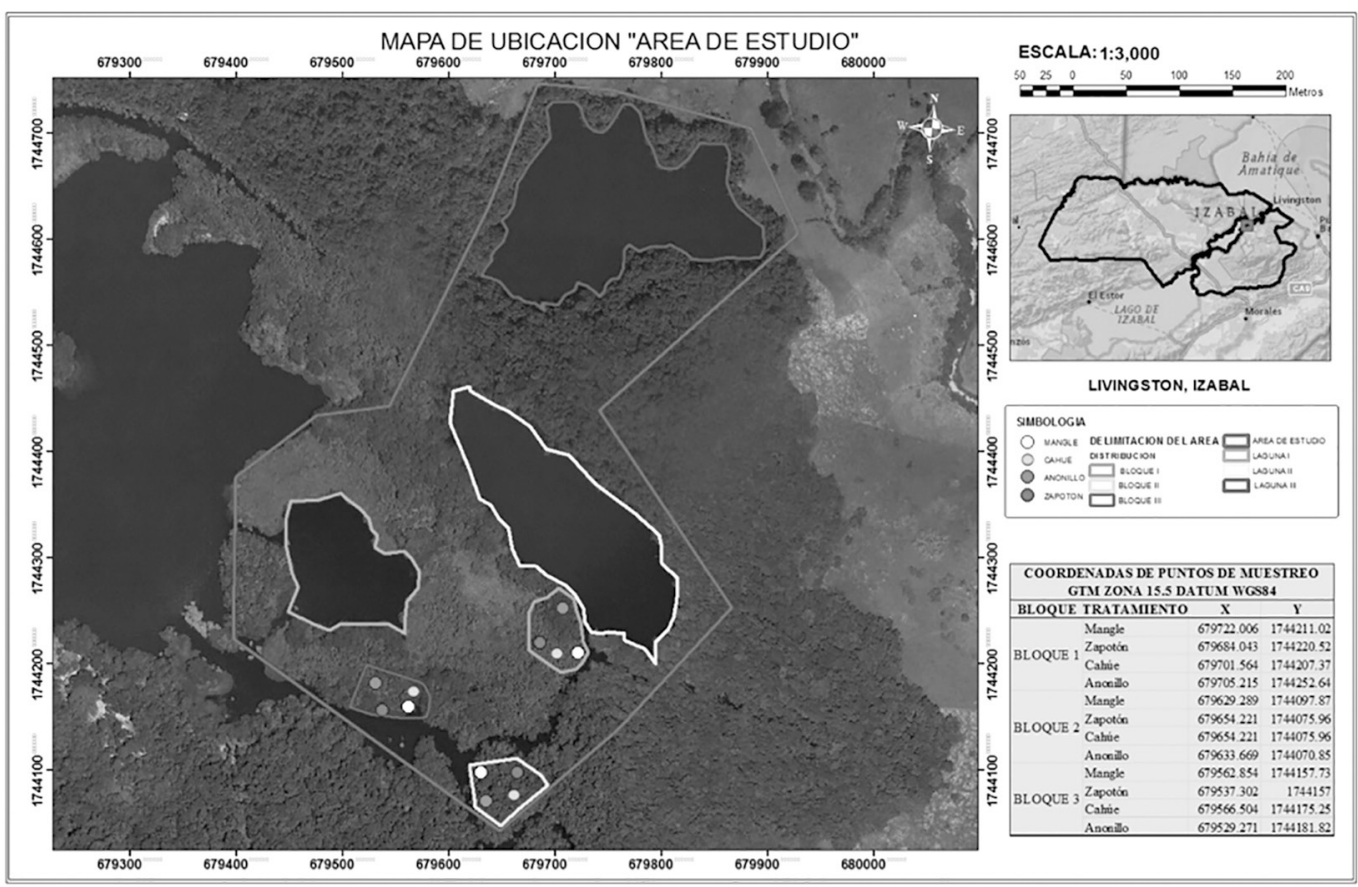

Fuente: Elaboración propia

\subsection{Colecta de las muestras de suelos}

Las parcelas, que a su vez se constituyen en repeticiones de cada tratamiento (área por especie vegetal), tienen una extensión de $100 \mathrm{~m}^{2}$, espacio en el que de forma aleatoria caminando en zigzag, se colectaron 6 submuestras durante el mes de fe- gura 1. Para ello se realizaron visitas de campo con objeto de corroborar los datos de gabinete contra la realidad de los escenarios en campo. Posteriormente se procedió a georreferenciar las parcelas donde se estableció el experimento; mismas que fueron dispuestas en bloques al azar. Para cada experimento se definen cuatro tratamientos (Sistema de mangle; áreas de Zapotón; Cahué y Anonillo), cada uno, con tres repeticiones. Donde la variable respuesta fue contenido de materia orgánica en el suelo (suelo sin raíces) a la profundidad $(0-30 \mathrm{~cm})$. 


\subsection{El análisis estadístico}

Los resultados de los análisis de laboratorio se realizaron con ayuda del software para análisis estadístico de aplicación general denominado InfoStat. El módulo estadístico que se utilizó fue diseño experimental en bloques completamente al azar. Este con cuatro tratamientos: Mangle rojo (Rizophora mangle), Zapatón (Pachira acuatica), Cahue (Pterocarpus officinalis), y Anonillo (Rollinia pittieri), y tres repeticiones, cada uno.

Modelo estadístico: $y i j=\mu+\mathrm{Ti}+\beta \mathrm{j}+$ Qij

Donde:

$\mu=$ media general

$\mathrm{Ti}=$ efecto del i-ésimo tratamiento

$\beta j$ = efecto del j-ésimo bloque

Qij $=$ error experimental en la unidad $\mathrm{j}$ del tratamiento $\mathrm{i}$

Qij $N I D(0, \sigma 2)$.

Modificado de (López Gonzáles, 2014).

Así mismo se realizó análisis de varianza para la media del contenido de materia orgánica de cada tratamiento para determinar, el efecto o no, de la especie vegetal sobre el contenido de materia orgánica en el suelo. Los efectos significativos fueron sometidos al test de igualdad de medias de Tukey con significancia del $5 \%$.

\subsection{Estimación del carbono capturado en el suelo}

Con base en el contenido de materia orgánica, se utilizó el factor de Bremmelen (0.58*M.O.) para obtener el carbono fijado en el suelo. De tal manera que conociendo la cantidad de hectáreas por especie vegetal; se estimó la cantidad de carbono almacenado en el suelo por unidad de área, para un estrato de profundidad $0.3 \mathrm{~m}$ y densidad del suelo de $0.5 \mathrm{Mg} / \mathrm{m}^{3}$ (BIOMARCC, SINAC \& GIZ, 2012), de la siguiente manera:

$\mathrm{MgC}=$ Área $(\mathrm{ha})^{\star}$ Densidad del suelo $\left(\mathrm{Mg} / \mathrm{m}^{3}\right)$ * Profundidad $(\mathrm{m})$ * (\% Carbono/100)

Donde $1 \mathrm{MgC}=3.67 \mathrm{MgCO}_{2}$.

\section{Resultados y discusión}

Los resultados del análisis de suelos se presentan en la Tabla 2, organizados por tratamiento, repetición y variables respuesta. 
Tabla 2

Análisis de suelos del bosque natural Cayo Quemado

\begin{tabular}{|c|c|c|c|c|c|c|}
\hline \multirow{3}{*}{ Tratamiento } & \multirow[b]{3}{*}{ Repetición } & \multirow{3}{*}{\multicolumn{2}{|c|}{$\frac{\mathrm{dS} / \mathrm{m}}{\mathrm{C} . \mathrm{E}}$}} & \multirow{3}{*}{$\frac{\%}{\text { M.O.S }}$} & \multirow{2}{*}{\multicolumn{2}{|c|}{$\mathrm{meq} / 100 \mathrm{gr}$}} \\
\hline & & & & & & \\
\hline & & & & & $\mathrm{N}$ & $\mathrm{Al}+\mathrm{H}$ \\
\hline \multirow[t]{3}{*}{ Mangle } & Mangle R1 & 5.4 & 10.55 & 46.39 & 1.5 & 0 \\
\hline & Mangle R2 & 5.1 & 1.51 & 34.96 & 1.34 & 0.1 \\
\hline & Mangle R3 & 5 & 1.08 & 45.05 & 1.39 & 0.1 \\
\hline \multirow[t]{3}{*}{ Cahué } & Cahué R1 & 5 & 1.12 & 62.53 & 2.43 & 0.1 \\
\hline & Cahué R2 & 5 & 0.69 & 61.18 & 2.32 & 0.1 \\
\hline & Cahué R3 & 5 & 1.95 & 76.65 & 2.74 & 0.1 \\
\hline \multirow[t]{3}{*}{ Anonillo } & Anonillo R1 & 5.3 & 4.54 & 59.17 & 1.62 & 0.2 \\
\hline & Anonillo R2 & 5.3 & 3.97 & 60.51 & 2.38 & 0.1 \\
\hline & Anonillo R3 & 4.4 & 0.42 & 77.99 & 2.05 & 0.3 \\
\hline \multirow[t]{3}{*}{ Zapotón } & Zapotón R1 & 4.4 & 0.51 & 68.58 & 1.55 & 0 \\
\hline & Zapotón R2 & 4.3 & 0.46 & 72.61 & 1.57 & 0.1 \\
\hline & Zapotón R3 & 5 & 2.18 & 71.27 & 1.94 & 0.1 \\
\hline
\end{tabular}

Fuente: Elaboración propia

dS/m= Conductividad Eléctrica; $\mathrm{pH}=$ Potencial Hidrógeno; M.O.S= Materia Orgánica del suelo; N= Nitrógeno; meq/100 gr= Capacidad Catiónica; $\mathrm{AL}+\mathrm{H}=$ Aluminio + Hidrógeno.

\section{El Potencial de Hidrógeno (pH)}

El $\mathrm{pH}$ del suelo se encuentra entre 4.3 y 5.4 , valores que categorizan a los suelos como ácidos, esto afecta la distribución de la fauna edáfica (lombrices y la biomasa bacteriana), son suelos empobrecidos de nutrientes (Mg, Ca, K y P), esto también afecta a las plantas en su nutrición de minerales ya que está estrechamente ligada a la acidez y la solubilidad de los nutrientes, e influye sobre la solubilidad de compuestos como (Al, Fe, Zn, Mn) que llegan a provocar efectos tóxicos para las plantas (Casanova, 2005).

\section{Conductividad Eléctrica (CE)}

Los resultados de la conductividad eléctrica, indican que el tratamiento mangle, repetición 1, está sobre suelo fuertemente salino; Anonillo Repetición 1 en moderadamente salino; siendo que las demás parcelas en suelos ligeramente salinos y no salinos. No se observa efecto de salinidad sobre parcelas según la clasificación de indicadores de la calidad del suelo de USDA (1999). Siendo que las parcelas experimentales más cercanas al canal del Río Dulce contienen mayor contenido de sales por influencia del intercambio del agua del río y el mar.

\section{Materia orgánica del suelo (M.O.S)}

Para los tratamientos Cahué; Anonillo y Zapotón el contenido de $\mathrm{MOS}$ en la fracción mineral del suelo a profundidad $(0-30 \mathrm{~cm})$ fue mayor del $60 \%$; es importante mencionar que se encuentran en partes más sólidas dentro de los Cayos, en cuanto que para el Mangle fue de $42 \%$. Esto coincide con las investigaciones de BIOMARCC, SINAC \& GIZ (2012) que indicaron que la concentración de carbono (\%) aumenta significativamente $(p<0.0001)$, entre $7.1 \%$ y 11.8 , a lo largo de la orilla del canal hacia tierra adentro del manglar, por transecto de $25 \mathrm{~m}$. Esto sugiere una mayor dinámica de sedimentación y acumulación de materia orgánica lejos del canal del río. 


\section{Interpretación del análisis estadístico}

Para determinar la correlación que existe entre las variables de respuesta, se utilizó la correlación de Pearson, que indicó correlación negativa entre $\mathrm{pH}$ y C.E (0.67) valor-p=0.016; entre $\mathrm{pH}$ y M.O (-0.58) valor-p $=0.048$; y positiva entre M.O y nitrógeno (0.563) valor- $\mathrm{p}=0.056$; independientemente del tratamiento y bloque, debido a que la acidez y condiciones de humedad limitan la descomposición de la materia orgánica a través de la mineralización., Tabla 3.

Tabla 3

Correlación de Pearson entre variables de respuesta

\begin{tabular}{lcrrrrr} 
& $\begin{array}{c}\text { Datos } \\
\text { tipificados }\end{array}$ & $\begin{array}{c}\text { Obs. Sin da- } \\
\text { tos perdidos }\end{array}$ & Mínimo & Máximo & Media & Desv. Típica \\
\hline pH & 12 & 12 & 4.300 & 5.400 & 4.933 & 0.37 \\
C.E & 12 & 12 & 429.500 & 10550.000 & 2418.167 & 2891.906 \\
\hline M.O & 12 & 12 & 34.960 & 77.990 & 61.408 & 13.421 \\
N & 12 & 12 & 1.340 & 2.740 & 1.903 & 0.473 \\
AL+H & 12 & 12 & 0.000 & 0.300 & 0.108 & 0.079
\end{tabular}

Matriz de correlaciones

\begin{tabular}{|c|c|c|c|c|c|}
\hline Variables & $\mathrm{pH}$ & C.E & M.O & $\mathrm{N}$ & $\mathrm{Al}+\mathrm{H}$ \\
\hline $\mathrm{pH}$ & 1 & 0.677 & -0.580 & 0.102 & -0.196 \\
\hline C.E & 0.677 & 1 & -0.379 & -0.181 & -0.320 \\
\hline M.O & -0.580 & -0.379 & 1 & 0.563 & 0.331 \\
\hline$N$ & 0.102 & -0.181 & 0.563 & 1 & 0.186 \\
\hline $\mathrm{Al}+\mathrm{H}$ & -0.196 & -0.320 & 0.331 & 0.186 & 1 \\
\hline
\end{tabular}

Valores -p

\begin{tabular}{|c|c|c|c|c|c|}
\hline Variable & $\mathrm{pH}$ & C.E & M.O & $\mathrm{N}$ & $\mathrm{Al}+\mathrm{H}$ \\
\hline $\mathrm{pH}$ & 0 & 0.016 & 0.048 & 0.752 & 0.541 \\
\hline C.E & 0.016 & 0 & 0.225 & 0.574 & 0.31 \\
\hline M.O & 0.048 & 0.225 & 0 & 0.056 & 0.293 \\
\hline$N$ & 0.752 & 0.574 & 0.056 & 0 & 0.563 \\
\hline $\mathrm{Al}+\mathrm{H}$ & 0.541 & 0.31 & 0.293 & 0.563 & 0 \\
\hline
\end{tabular}

Fuente: Elaboración propia

Análisis de varianza

El análisis de varianza del contenido de materia orgánica en el suelo, indica que hay efecto de tratamiento sobre la variable respuesta, es decir, que estadísticamente hay diferencias del efecto de las especies vegetales en Cayo Quemado sobre el almacenamiento de carbono en el suelo a una significancia del $1 \%$, Tabla 4. 
Tabla 4

Análisis de varianza del contenido de materia orgánica en el suelo

\begin{tabular}{lrrrrr}
\multicolumn{1}{c}{ Variable } & $\mathrm{N}$ & $\mathrm{R}$ & $\mathrm{R} \mathrm{R}^{2} \mathrm{Aj}$ & $\mathrm{CV}$ \\
\hline M.O & 12 & 0.77 & 0.69 & 12.27 \\
$\begin{array}{l}\text { Cuadro de análisis de } \\
\text { varianza (SC Tipo III) }\end{array}$ & & & & & \\
\hline F.V. & $\mathrm{SC}$ & $\mathrm{gl}$ & $\mathrm{CM}$ & $\mathrm{F}$ & $\mathrm{P}$-valor \\
\hline Modelo. & 1527.35 & 3 & 509.12 & 8.97 & 0.0061 \\
Tratamiento & 1527.35 & 3 & 509.12 & 8.97 & 0.0061 \\
\hline Error & 453.86 & 8 & 56.73 & & \\
\hline Total & 1981.21 & 11 & & & \\
\hline
\end{tabular}

Fuente: Elaboración propia.

Debido al efecto significativo entre tratamientos, se realizó prueba de diferencia entre medias de Tukey, donde se determinó que efectivamente el Mangle almacena menor cantidad de materia or- gánica en el suelo en relación al Cahué, Anonillo y Zapotón, no existiendo diferencia estadística entre estas últimas tres, Tabla 5 .

Tabla 5

Prueba de Tukey para diferencia del contenido medio de materia orgánica por tratamiento

\begin{tabular}{lcccc}
\hline Test: Tukey Alfa=0.05 DMS $=19.69423$ & & & & \\
\hline Error: 56.7324 gl: 8 & Medias & n & E.E. & \\
\hline Tratamiento & 42.13 & 3 & 3.39 & A \\
\hline Mangle & 65.89 & 3 & 3.39 & B \\
\hline Anonillo & 66.79 & 3 & 3.39 & B \\
Cahué & 70.82 & 3 & 3.39 & B \\
\hline Zapotón & & & & \\
\hline
\end{tabular}

Medias con una letra común no son significativamente diferentes ( $p>0.05)$.

Fuente: Elaboración propia.

Carbono almacenado en el suelo

Cayo Quemado tiene la capacidad de almacenar en la parte mineral del suelo $0.5164 \mathrm{MgC}$; equi- valente a $1.8959 \mathrm{Mg} \mathrm{CO}_{2}$ en 10.56 de sus 12.69 ha; como efecto de las cuatro especies vegetales dominantes en dicha área, Tabla 6. 
Tabla 6

Carbono almacenado en el suelo sin raíces por especie vegetal

\begin{tabular}{lcccccc} 
Tratamiento & MOS \% & C\%=MOS*0.58 & MgC/ha & $\begin{array}{c}\text { Área (ha) por } \\
\text { especie vegetal }\end{array}$ & $\begin{array}{c}\text { MgC/ha por } \\
\text { especie vegetal en } \\
\text { el Cayo }\end{array}$ & $\begin{array}{c}\mathrm{Mg} \mathrm{CO}_{2} / \text { ha } \\
\text { por especie } \\
\text { vegetal en el Cayo }\end{array}$ \\
\hline Mangle & 42.13 & 24.4354 & 0.0366 & 4.89 & 0.1792 & 0.6576 \\
Cahué & 66.78 & 38.7324 & 0.0580 & 2.35 & 0.1365 & 0.5009 \\
Anonillo & 65.89 & 38.2162 & 0.0573 & 0.91 & 0.0521 & 0.1912 \\
Zapotón & 70.82 & 41.0756 & 0.0616 & 2.41 & 0.1484 & 0.5446 \\
\hline Total & & & & 10.56 & 0.5164 & 1.8951
\end{tabular}

Fuente: Elaboración propia

Por estos y otros servicios ecosistémicos, se ha propuesto incorporar a los ecosistemas marino-costeros como elementos de las iniciativas internacionales de mitigación al cambio climático. Sin embargo, aun la dinámica de pérdidas y ganancias de carbono en los diferentes ecosistemas no está clara, más aun, en Centroamérica la información acerca de la dinámica y captura de carbono en manglares, pastos marinos y marismas saladas es limitada (BIOMARCC-SINAC-GIZ, 2012), y en el caso de Guatemala todavía no existe una política sobre el incentivo a la captura de carbono en áreas marino costeras.

\section{Conclusiones}

En el bosque natural Cayo Quemado, las especies forestales asociadas juegan un papel importante en cuanto al anclaje y salud del suelo; situación que se refleja a través de los contenidos de materia orgánica en las parcelas ubicadas a mayor distancia del canal del Río Dulce hacia tierra dentro del Cayo.

Existe efecto estadísticamente significativo de la especie vegetal sobre el almacenamiento de carbono en el suelo, donde el sistema manglar almacena menor cantidad de carbono en relación con el Cahué, Anonillo y Zapotón; no habiendo diferencia estadística entre estas últimas tres.

Cayo Quemado tiene capacidad de almacenar en el suelo sin raíces $1.89 \mathrm{Mg} \mathrm{CO}_{2}$ en 10.56 ha de bosque natural.

\section{Referencias bibliográficas}

Adame, M. et al. (2013). "Carbon stocks of tropical coastal wetlands with in the karstic landscape of the Mexican Caribbean". PLoS ONE, 8.

Aguilera, S. (2000). "Importancia de la protección de la materia orgánica en suelos". En: Simposio Proyecto Ley Protección de Suelo. Boletín No 14. Valdivia (Chile): pp. 77-85.

BIOMARCC-SINAC-GIZ. (2012). Evaluación de carbono en el Humedal Nacional Térraba-Sierpe. San José (Costa Rica): BIOMARCC-SINAC-GIZ

Blanco, J., Ortiz, L. y Urrego, L. (2015). "Reservorios de biomasa aérea y de carbono en los manglares del golfo de Urabá (Caribe colombiano)". Actualidades Biológicas, pp. 131-141.

Casanova, E. (2005). Introducción a la ciencia del suelo. Universidad Central de Venezuela. Consejo de desarrollo científico y humanístico. Caracas.

Castellanos, M. y J. Menjivar (2011). "Tensores edáficos de los manglares de La Guajira". En: Tierra en el pensamiento, pequeñas acciones para medir la salud del planeta. D. C: Grupo de investigación territorios Semiáridos del Caribe, Universidad de La Guajira. pp. 39-42 
CONAP. (2011). Plan maestro refugio de vida silvestre Punta de Manabique. (Guatemala). Consejo Nacional de Áreas Protegidas.

CONAP. (2015). Plan Maestro Parque Nacional Río Dulce. (2da. ed). Izabal (Guatemala): Consejo Nacional de Áreas Protegidas.

(2015). Plan maestro Parque Nacional Río Dulce (PNRD). Segunda Actualización. (Guatemala): CONAP-FONACON.

CONAP-INAB. (2014). Plan de Manejo para bosques naturales con fines de protección. Livingston, (Guatemala): CONAP-INAB.

Donato, D., et al (2011). "Mangroves among the most carbon-richforests in the tropics". Nature Geoscience, pp. 293-297.

FAO. (2007). The World's Mangroves 1980-2005: A thematic study prepared in the framework of the Global Forest Resources Assessment 2005. Roma (Italia):

Galantini, J. (2002). "Contenido y calidad de las fracciones orgánicas del suelo bajo rotaciones con trigo en la región semiárida pampeana". INTA, pp. 125-146.

Jiménez, J. (1999). "Ambiente, distribución y características estructurales en los Manglares del Pacífico de Centro América: Contrastes climáticos". En: A. Yáñez-Arancibia y A. L. Lara-Domínguez (Eds.). Ecosistemas de Manglar en América Tropical. Instituto de Ecología A.C. México, UICN/ORMA, Costa Rica, NOAA/ NMFS Silver Spring MD USA. pp. 51-70.

Lacerda, L., et al. (2001) Mangrove ecosystem, function and management. Springer. In: L.D. Lacerda (Ed.) Berlín (Alemania): American Mangroves.

Lal, R., (1997). "Residue management, conservation tillage and soil restoration for mitigating greenhouse effect by $\mathrm{CO}_{2}$ - enrichment". Soil Till. Res, pp. 81-107.
López, E. y B. González, B. (2014). Diseño y Análisis de Experimentos. (Guatemala): FAUSAC/Universidad de San Carlos de Guatemala.

Lozano, Y. (2007). "Los sumideros de Carbono: Un análisis de la potencialidad económica en un bosque de manglar del Pacífico Colombiano". Revista EIDENAR, pp. 82-92.

Lugo, A., Mediana, E. y McGinley, K. (2014). "Issues and Challenges of Mangrove conservation in the Anthropocene". Madera y Bosques, pp 11-38.

MARN. (2013). Informe técnico estudio de la cobertura de mangle en la República de Guatemala. Guatemala. Ministerio de Ambiente y Recursos Naturales

Martel, C. y L. Cairampoma. (2012). "Cuantificación del carbono almacenado en formaciones vegetales amazónicas en Cicra, Madre De Dios (Perú). Ecología Aplicada, pp. 59-65.

Martínez, E., Fuente, J. y Acevedo, E. (2008). "Carbono orgánico y propiedades del suelo". R.C. Suelo Nutr. Veg., pp. 68-96

Murdiyarso, D. et al, (2009). Carbon storage in mangrove and peatland ecosystems: A preliminary account from plots in Indonesia. Bogor Barat, (Indonesia): Center for International Forestry Research Center for International Forestry Research CIFOR.

Ospina, J. (2010). Manual de semillas de árboles tropicales Pachira aquatica Aubl. En J. A. Vosso (Ed.), Departamento de Agricultura de los Estados Unidos, Servicio Forestal. pp. 583-661.

PNUMA. (2013). Manejo integrado de las zonas costeras y gestión sostenible de los manglares en Guatemala, Honduras y Nicaragua. (Honduras).

Saenger, P. (2002). Mangrove ecology, silviculture and conservation. Kluwer Academic Publishers, Dordrecht, The Netherlands. 
Saint-Paul, U. (2006). "Interrelations among mangroves, the local economy and social sustainability: a review from a case study in north Brazil". In: C.T. Hoanh, T.P. Tuong, J.W. Gowing, and B. Hardy, eds. Environment and Livelihoods in Tropical Coastal Zones, CAB International: Wallingford, UK.

Schlesinger, W. y J. Andrews. (2000). Soil respiration and the global carbon cycle. Biogeochemistry.

Simmons, Ch., Tarano, J. y Pinto J. (1959). Clasificación de Reconocimiento de los Suelos de la República de Guatemala. Instituto Agropecuario Nacional. Servicio Cooperativo Inter-Americano de Agricultura, Ministerio de Agricultura. Guatemala.

USDA. (1999). Guía para la Evaluación de la Calidad y Salud del Suelo. Departamento de Agricultura de los Estados Unidos. Estados Unidos.
USDA. (2014). Claves para la Taxonomía de Suelos. (12a. Ed.). Departamento de Agricultura de los Estados Unidos. Estados Unidos.

URL-IARNA. (2012). Perfil Ambiental de Guatemala (2010-2012). Vulnerabilidad local y creciente construcción de riesgo. (Guatemala): Universidad Rafael Landívar-Instituto de Agricultura, Recursos Naturales y Ambiente.

Wilkie, M. L. y S. Fortuna (2003) "Status and Trends in Mangrove Area Extent Worldwide". Forest Resources Assessment Working paper, no. 63. Forest Resources Division. (Sin publicar) Disponible en línea en: http://www.fao.org/docrep/007/j1533e/j1533e00.htm, FAO. Roma.

Zamora, N. (2011). Manual de Plantas de Costa Rica. Instituto Nacional de Biodiversidad. Costa Rica. 
